\title{
A nonhuman primate model of chikungunya disease
}

\author{
Stephen Higgs and Sarah A. Ziegler
}

Department of Pathology, University of Texas Medical Branch, Galveston.

\begin{abstract}
Chikungunya disease is a severely debilitating, mosquito-borne, viral illness that has reached epidemic proportions in Africa, Asia, and the islands of the Indian Ocean. A mutation enhancing the ability of the chikungunya virus (CHIKV) to infect and be transmitted by Aedes albopictus has increased the geographical range at risk for infection due to the continuing global spread of this mosquito. Research into disease pathogenesis, vaccine development, and therapeutic design has been hindered by the lack of appropriate animal models of this disease. The meticulous study reported in this issue of the JCI by Labadie et al. is one of the first reports describing CHIKV infection of adult immunocompetent nonhuman primates. Using traditional and modern molecular and immunological approaches, the authors demonstrate that macaques infected with CHIKV are a good model of human CHIKV infection and also show that persistent arthralgia in humans may be caused by persistent CHIKV infection of macrophages.
\end{abstract}

Arthrogenic alphaviruses are widely distributed. For example, Ross River virus (RRV) affects thousands every year in Australia, causing polyarthritis disease; Mayaro virus persists in the Amazon rainforest, causing small, sporadic outbreaks; and O'nyong-nyong virus is known to cause explosive outbreaks of thousands of people in central Africa. However, the arthrogenic alphavirus that has become most well known in recent years is chikungunya virus (CHIKV), the subject of the study by Labadie et al. in this issue of the JCI (1).

The comprehensive review "Chikungunya virus disease," published in volume II of Tom Monath's landmark series The arboviruses: epidemiology and ecology (2), has remained the classic reference for this virus for more than 20 years. However, after the 2005-2006 CHIKV epidemic in the Indian Ocean Islands, several excellent reviews have been published (3-5). CHIKV was first isolated in Tanzania in 1952, during a severe dengue fever-like epidemic (6). However, chikungunya fever was likely confused with dengue for hundreds of years, with the first possible record of chikungunya fever published in Cairo in 1779 (7). It has been speculated that CHIKV was introduced into the Americas

Conflict of interest: The authors have declared that no conflict of interest exists.

Citation for this article: JClin Invest. 2010; 120(3):657-660. doi:10.1172/JCI42392. in the early 1800s, but failed to establish a sustainable transmission cycle (8).

Since 1952, laboratory-confirmed outbreaks of chikungunya fever have occurred almost annually in south and central Africa and southeast Asia (2, 4). Between 1990 and 1996, very few outbreaks of chikungunya fever were reported, except in Thailand. Chikungunya fever outbreaks became more frequent and widespread, starting in 2003 in Indonesia. In 2005-2006, the islands of the Indian Ocean, including Seychelles, Mauritius, Madagascar, and La Réunion, were devastated by widespread epidemics of CHIKV infection (4). A chikungunya fever epidemic occurred in India during 2006-2007, where it is estimated that more than 1.4 million people were infected. In July 2007, CHIKV emerged from the tropics and caused a localized outbreak in Ravenna, Italy (9). In 2008-2009, CHIKV was reported in countries of southeast Asia, including India, Thailand, and Singapore. The epidemic continues at the time of this writing, with an estimated 12,000 cases in Indonesia's Lampung province reported between midDecember 2009 and mid-January 2010.

\section{Clinical presentation of CHIKV infection}

CHIKV infection in humans usually causes a nonlethal, self-limiting, febrile illness. Until recently, much of our knowledge of human disease has been descriptive. Patients developed viremias that could exceed $10^{9}$ mouse
$\mathrm{LD}_{50} / \mathrm{ml}$ and last for 4 days, and displayed nonfatal symptoms of variable severity that could persist for many months $(10,11)$. Chikungunya fever is usually characterized by high fever, arthralgia, and rash. During the acute phase, patients can experience painful and disabling arthritis that can last for 7 days (12). During some outbreaks, twothirds of patients have had to be hospitalized (12). Unfortunately, after the acute phase, polyarthritis can be recurrent and may persist for up to several years after infection (4). Other symptoms of CHIKV infection can include retro-orbital pain, neurological and hemorrhagic manifestations, and myocarditis. In the most recent epidemics, many nontypical symptoms were reported for the first time, including lethal hepatitis, encephalitis, maternal-fetal transmission, and an increased death rate (4).

The epidemic infecting approximately 300,000 people on the island of La Réunion (a French territory popular with tourists), the importation of cases to many other countries, and establishment of a locally transmitted epidemic in Italy was the impetus for multiple governments to provide a considerable infusion of funding to stimulate major research efforts to improve our understanding of this hitherto largely ignored disease. The apparently increased severity of infection (with reports of approximately 250 fatalities during the Indian Ocean epidemic; ref. 1) may be attributed to several factors, for example, naivety of the afflicted population, preexisting medical conditions, and better reporting of the illness in a modern health care setting. The well-documented A226V mutation in CHIKV, which has arisen independently in several locations, does not seem to be correlated with disease severity, although it has been proven to influence infectivity for the Asian tiger mosquito (Aedes albopictus; refs. 13-16). This adaptation is a cause for concern, as this highly invasive and relatively cold-tolerant mosquito is gradually spreading to new areas in Europe and elsewhere (17). Some climate change proponents have suggested that recent chikungunya fever epidemics might be climate 
Human CHIKV infection (natural infection by mosquito)

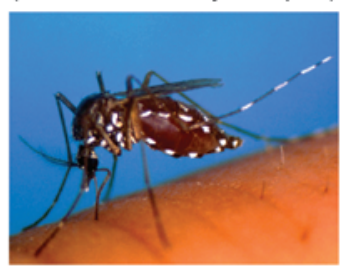

Primate CHIKV infection (laboratory infection by needle)

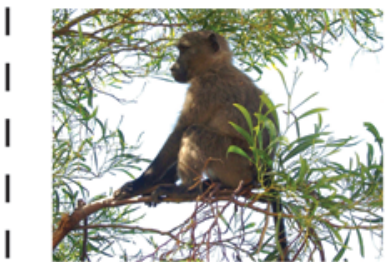

Acute phase

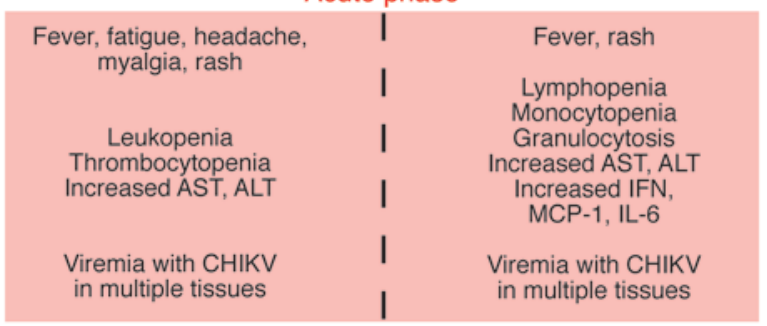

Subacute phase

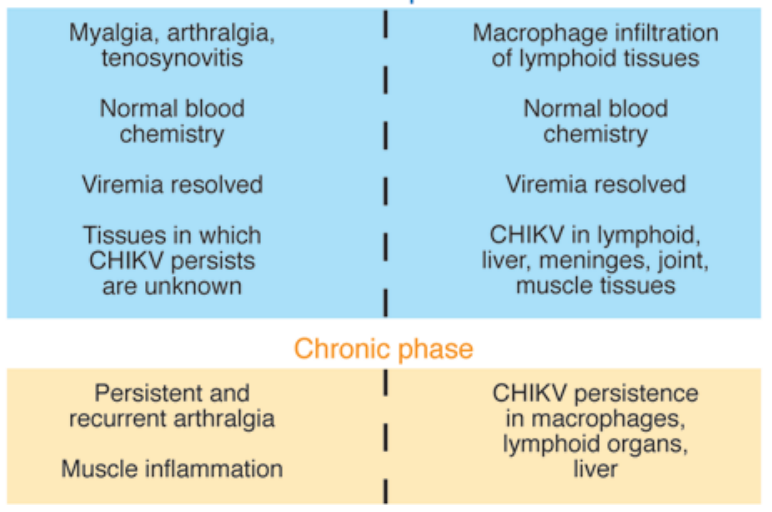

\section{Figure 1}

Comparison of symptoms resulting from naturally acquired human $\mathrm{CHIKV}$ infection and observed symptoms in a laboratory-infected nonhuman primate model of chikungunya disease. In their study in this issue of the $\mathrm{JCl}$, Labadie et al. describe the stages of symptoms of CHIKV infection seen in their primate model as having 3 phases: acute, subacute, and chronic (1). These symptoms may be correlated with recently published observations of the symptoms in human cases of chikungunya fever $(4,5,12,23)$. In the acute phase, nonhuman primates show high viremia (approximately $10^{8}-10^{9}$ viral RNA copies/ml), fever, and rash. They also have increased levels of aminotransferase, including aspartate transaminase (AST) and alanine transaminase (ALT), as well as increased expression of IFN, MCP-1, and IL-6. The nonhuman primates also have abnormal blood chemistries, including lymphopenia, monocytopenia, and granulocytosis. Humans in the acute phase of chikungunya fever present with fever, fatigue, headache, myalgia, and rash as well as increased AST and ALT. Viremia in humans ranges from $10^{3}$ to $10^{10}$ viral RNA copies/ml. Leukopenia and thrombocytopenia are common. During the subacute phase in nonhuman primates, there is macrophage infiltration, and CHIKV is present in lymphoid tissue, liver, meninges, joints, and muscle. The subacute phase is not well characterized in humans, but includes the persistence of myalgia, arthralgia, and tenosynovitis. In the chronic phase in nonhuman primates, there was CHIKV persistence in macrophages, lymphoid tissue, and liver tissue up to 2 months after infection. The chronic phase in humans includes persistent and recurrent arthralgia that can last for months to years. associated, but no data support the suggestion (18). Global travel and commercialization are likely contributors through rapid movement of infected humans and vector invasions, respectively.

\section{Mouse models of CHIKV and RRV}

As with dengue fever, the lack of a good animal model replicating human chikungunya fever symptoms and pathology has been a major obstacle for understanding the course of infection, spectrum of disease severity, and persistence of symptoms. A mouse model for RRV, predating the recent work with CHIKV, is characterized by hind limb dragging due to the destruction of the skeletal muscle (19). Multiple CHIKV mouse models have been proposed, but they are either unable to provide a good working background for vaccine-related testing or unsuccessful in reproducing true human-like symptoms. Subcutaneous injections of CHIKV in young outbred mice, although they induce disease similar in symptomology to CHIKV infection in humans, are not good platforms for the study of vaccine efficacy (20). Intrana- sal inoculation of CHIKV into inbred mice results in neurological involvement and high mortality (21), symptoms not seen in human CHIKV infection. Other models using immunodeficient mice, including IFN-deficient mice (22), partially mimic human disease, but are not good models for vaccine testing.

\section{Primate models of CHIKV}

The current study reported by Labadie et al. incorporates both traditional and stateof-the-art molecular and immunological approaches to evaluate a nonhuman primate model for this important, emerging, vector-borne disease - specifically, infection of long-tailed macaques (Macaca fascicularis) with a CHIKV isolate from a patient infected during the recent La Réunion outbreak (1). The symptoms and pathology observed in the nonhuman primate model resembled many of those previously reported for CHIKV infection in humans, and so the data are a significant contribution to our understanding of the disease (Figure 1 and refs. 5, 23). It is gratifying that the study was not driven by the use of highly sensitive molecular techniques that do not distinguish between infectious and defective virus, but incorporated more traditional infectivity and immunological assays that can be readily interpreted by a broad audience in the context of previous work. The synergism of using multiple techniques and the duration of the study has begun to explain the mechanisms underlying the long-term persistence of chikungunya fever symptoms. The reported correlation between dose and disease severity (more severe at a higher dose) is interesting and may explain, at least in part, the range of symptoms observed in humans. The doses used by the authors are within ranges likely delivered by mosquitoes (24). It would have been interesting if some primates had been challenged by $\mathrm{CHIKV}$-infected mosquitoes, given the increasing evidence that chikungunya fever has an immunological component. Mosquito saliva is known to have immunomodulatory activity, and, for several viruses (for example, West Nile virus), virus delivery in the context of saliva can influence infection establishment, dissemination, and severity $(25,26)$. As Labadie et al. state, the 
macaque model should help in the development of new therapeutic or prophylactic strategies against chikungunya fever and facilitate testing of new interventions (1). This work represents a considerable advance over early primate studies $(27,28)$, and development of a nonhuman primate model of CHIKV infection has been a focus of several groups. The in utero infections observed during the La Réunion epidemic have been evaluated in a macaque model (29). Importantly, a macaque model has been developed at the NIH laboratories to determine the efficacy of a much-needed CHIKV vaccine and has demonstrated excellent protection against challenge with virulent CHIKV (30). This model, like that reported here by Labadie et al., mimicked key features of human chikungunya disease.

Labadie et al.'s current work in macaques (1) shows a good correlation with previously published work with mice. With little neurological involvement and virus prevalence in immune tissues and muscles, the present model shows promise. The authors admit that a limitation of their model is the lack of joint and muscle pathology in the macaques, but note that the occurrence of these symptoms in humans is restricted to the most severe cases, although recent reports have stated that arthralgia/arthritis actually affects $73 \%-80 \%$ of patients (5). Another limitation of the Labadie et al. paper is that no serology studies were done. It would be interesting to see the level and types of antibodies that the macaques produced in response to CHIKV infection.

\section{Immunopathology during CHIKV infection}

Labadie et al. present a compelling argument for the importance of macrophages in causing CHIKV-induced disease (1). Macrophages have been shown to be one of the keys to the destructive muscle necrosis, and possibly viral persistence, seen in RRV infection in mice. These early observations of RRV infection in mice are substantiated in the work by Labadie et al. Rulli et al. discussed the recent RRV data showing that macrophages are a key mediator of muscle necrosis and pathology and that, in vitro, RRV can persistently infect macrophages and upregulate release of IFN- $\gamma$, TNF- $\gamma$, and monocyte chemoattractant protein-1 (MCP-1; ref. 31). Macrophage infiltration into muscles and joints in RRV-infected mice was responsible for the production of cytokines and chemokines, such as TNF- $\alpha$, macrophage inflammatory protein-1 $\alpha$ (MIP-1 $\alpha$ ), MCP-1,
IL-1 $\beta$, and IFN- $\gamma$ (19). In the CHIKV study by Labadie et al., infected macaques showed increased expression of IFN- $\alpha / \beta$, CCL2, CCL3, CCL4, IL- 6 , and IFN- $\gamma$ and moderate increases of TNF- $\alpha$ (1). These findings are what would be expected of a viral infection inducing a strong macrophage response. The typical antiviral response in humans is characterized by the induction of cytokines such as IFN- $\alpha / \beta$, IFN- $\gamma$, IL-12, and IL-18 and the activation of macrophages, NK cells, dendritic cells, neutrophils, and complement. TNF, IL-1, and IL- 6 are produced by immune cells as signals of inflammation. Cytokines IL-4 and IL-10, which are not upregulated during viral infection, were not upregulated in the current study (1), again emphasizing the importance of macrophages.

\section{Wild primates and CHIKV transmission cycles}

The demonstration of $M$. fascicularis susceptibility to CHIKV infection (1) raises an interesting epidemiological question that merits further investigation. Within what can be considered as the endemic/original geographical region of Africa in which CHIKV circulates, primates have been implicated in a transmission cycle that does not involve humans. McIntosh et al. (32) reported CHIKV seropositive, wild-ranging vervets (Ceropithecus aethiops pygerythrus) and baboons (Papio ursius) collected in the 1962 endemic area of southern Rhodesia and experimentally recorded that viremias in these animals lasted for 3-4 days in both species, "at levels adequate to infect significant numbers of susceptible mosquitoes" (33). In Asia, the potential involvement of primates in the transmission cycle is unclear. Inoue et al. reported an IgM seropositive rate of $14.8 \%$ for $M$. fascicularis in the Philippines (34), and Marchette et al. reported seropositive monkeys in Malaya - although 15 of 16 were also positive for other related viruses, which can compromise the tests' accuracy (35). However, Peiris et al. found no CHIKV positivity among 115 wild toque macaques (Macaca sinica) from Sri Lanka (36). The suggestion that there is a sylvatic cycle in Asia must be treated with caution, since evidence of infection in a wild animal must not be interpreted as proof of involvement in a transmission cycle. Specific criteria must be met when considering whether a vertebrate is involved in virus transmission. Basically, the vertebrate must be spatially and temporally coincident with a known vector, be naturally fed upon by that vector, and produce viremias of sufficient magnitude and duration to infect the vector.
Finally, transmission between the vertebrate and vector must be experimentally demonstrated. To date, these criteria have not been satisfied for primates in Asia, so we cannot assume that primates play any significant role in the Asian CHIKV transmission cycle. This is a gap in our knowledge that needs to be filled, and one to which the study by Labadie et al. contributes (1). M. fascicularis is widespread throughout Asia (37) and has been introduced into Hong Kong, Indonesia, Mauritius, and Palau. It is interesting that the species is present in large numbers on Mauritius (30,000 estimated; ref. 38), but there are no compelling data to implicate its involvement in the large chikungunya fever epidemic in the region (C. Chastel, personal communication).

Overall, the development and characterization of primate models for chikungunya fever represent a significant advance in the field that has already increased our understanding of the disease and facilitated evaluation of new potential vaccines. It is hoped that identification of the underlying mechanisms of the disease will be translated into a reduction of the consequences of infection and new preventive strategies that ultimately reduce disease incidence.

\section{Acknowledgments}

S. Higgs is supported in part by NIH grant R21 AI073389. S. Ziegler is supported by a fellowship from the Sealy Center for Vaccine Development.

Address correspondence to: Stephen Higgs, Department of Pathology, 2.138E Keiller Building, University of Texas Medical Branch, 301 University Blvd., Galveston, TX 77555-0609. Phone: 409.747.2426; Fax: 409.747.2437; E-mail: sthiggs@utmb.edu.

1. Labadie $\mathrm{K}$, et al. Chikungunya disease in nonhuman primates involves long-term viral persistence in macrophages. J Clin Invest. 2010;120(3):894-906.

2. Jupp PG, McIntosh BM. Chikungunya virus disease. In: Monath TP, ed. The Arboviruses: Epidemiology and Ecology. Boca Raton: CRC Press; 1998:137-157.

3. Charrel RN, de Lamballerie X, Raoult D. Chikungunya outbreaks--the globalization of vectorborne diseases. NEngl J Med. 2007;356(8):769-771.

4. Powers AM, Logue CH. Changing patterns of chikungunya virus: re-emergence of a zoonotic arbovirus. J Gen Virol. 2007;88(Pt 9):2363-2377.

5. Pialoux G, Gauzere BA, Jaureguiberry S, Strobel M. Chikungunya, an epidemic arbovirosis. Lancet Infect Dis. 2007;7(5):319-327.

6. Robinson MC. An epidemic of virus disease in Southern Province, Tanganyika Territory, in 195253. I. Clinical features. Trans R Soc Trop Med Hyg. 1955; 49(1):28-32.

7. Carey DE. Chikungunya and dengue: a case of mistaken identity? J Hist Med Allied Sci. 1971; 26(3):243-262. 
8. Halstead SB. Chikungunya. In: Feign RD, Cherry J, Demmler-Harrison GJ, Kaplan SL, eds. Feign and Cherry's Textbook of Pediatric Infectious Diseases. Philadelphia, PA: Saunders Elsevier; 2004:2178-2184.

9. Enserink M. Epidemiology. Tropical disease follows mosquitoes to Europe. Science. 2007;317(5844):1485.

10. Myers RM, Carey DE, Reuben R, Jesudass ES, De Ranitz C, Jadhav M. The 1964 epidemic of denguelike fever in South India: isolation of chikungunya virus from human sera and from mosquitoes. Indian J Med Res. 1965;53(8):694-701.

11. Anderson CR, Singh KR, Sarkar JK. Isolation of chikungunya virus from Aedes aegypti fed on naturally infected humans in Calcutta. Curr Sci. 1965;34:579-580.

12. Simon F, et al. Chikungunya infection; an emerging rheumatism among travelers returned from Indian Ocean Islands. Report of 47 cases. Medicine. 2007;86(3):123-137.

13. Schuffenecker I, et al. Genome microevolution of chikungunya viruses causing the Indian Ocean outbreak. PLoS Med. 2006;3(7):e263.

14. de Lamballerie X, Leroy E, Charrel RN, Tsetsarkin K, Higgs S, Gould EA. Chikungunya virus adapts to tiger mosquito via evolutionary convergence: a sign of things to come? Virol J. 2008;5:33.

15. Tsetsarkin KA, Vanlandingham DL, McGee CE, Higgs $S$. A single mutation in chikungunya virus affects vector specificity and epidemic potential. PLoS Pathog. 2007;3(12):e201.

16. Tsetsarkin KA, McGee CE, Volk SM, Vanlandingham DL, Weaver SC, Higgs S. Epistatic roles of E2 glycoprotein mutations in adaption of chikungunya virus to Aedes albopictus and Ae. aegypti mosquitoes. PLoS One. 2009;4(8):e6835.

17. Benedict MQ, Levine RS, Hawley WA, Lounibos LP. Spread of the tiger: global risk of invasion by the mosquito Aedes albopictus. Vector Borne Zoonotic Dis. 2007;7(1):76-85.

18. Gould EA, Higgs S. Impact of climate change and other factors on emerging arbovirus diseases. Trans R Soc Trop Med Hyg. 2009;103(2):109-121.

19. Lidbury BA, et al. Macrophage-derived proinflammatory factors contribute to the development of arthritis and myositis after infection with an arthrogenic alphavirus. J Infect Dis. 2008;197(11):1585-1593.

20. Ziegler SA, Lu L, da Rosa AP, Xiao SY, Tesh RB. An animal model for studying the pathogenesis of chikungunya virus infection. Am J Trop Med Hyg. 2008;79(1):133-139.

21. Wang $E$, et al. Chimeric alphavirus vaccine candidates for chikungunya. Vaccine. 2008;26(39):5030-5039.

22. Couderc T, et al. A mouse model for Chikungunya: young age and inefficient type-I interferon signaling are risk factors for severe disease. PLoS Pathog. 2008;4(2):e29.

23. Taubitz W, et al. Chikungunya fever in travelers: clinical presentation and course. Clin Infect Dis. 2007;45(1):e1-e4

24. Dubrulle M, Mousson L, Moutailler S, Vazeille M, Failloux AB. Chikungunya virus and Aedes mosquitoes: saliva is infectious as soon as two days after oral infection. PLoS One. 2009;4(6):e5895.

25. Boppana VD, Thangamani S, Adler AJ, Wikel SK. SAAG-4 is a novel mosquito salivary protein that programmes host CD4 T cells to express IL-4. Parasite Immunol. 2009;31(6):287-295.

26. Schneider BS, Soong L, Girard YA, Campbell G, Mason P, Higgs S. Potentiation of West Nile encephalitis by mosquito feeding. Viral Immunol. 2006;19(1):74-82.

27. Binn LN, Harrison VR, Randall R. Patterns of viremia and antibody observed in rhesus monkeys inoculated with chikungunya and other serologically related group A arboviruses. Am J Trop Med Hyg. 1967;16(6):782-785.

28. Paul SD, Singh KR. Experimental infection of Macaca radiata with Chikungunya virus and transmission of virus by mosquitoes. Indian J Med Res. 1968;56(6):802-811.

29. Chen C-I, et al. Development of a macaque model for in utero chikungunya virus infection. In: American Society of Tropical Medicine and Hygiene 58th Annual Meeting; November 18-22, 2009:Washington, DC. Abstract 2997.

30. Akahata W, et al. A virus-like particle vaccine for epidemic Chikungunya virus protects nonhuman primates against infection [published online ahead of print January 28, 2010]. Nat Med. doi: $10.1038 / \mathrm{nm} .2105$.

31. Rulli NE, et al. Ross River virus: molecular and cellular aspects of disease pathogenesis. Pharmacol Ther. 2005;107(3):329-342.

32. McIntosh BM, Peterson HE, McGillivray GM, de Sousa J. Further studies on the chikungunya outbreak in southern Rhodesia in 1962. I. Mosquitoes, wild primates and birds in relation to the epidemic. Ann Trop Med Parasitol. 1964;58:45-51.

33. McIntosh BM, Harwin RM, Paterson HE, Westwater ML. An epidemic of Chikungunya in South-Eastern Southern Rhodesia. Cent Afr J Med. 1963;43:351-359.

34. Inoue $\mathrm{S}$, et al. Distribution of three arbovirus antibodies among monkeys (Macaca fascicularis) in the Philippines. J Med Primatol. 2003;32(2):89-94.

35. Marchette NJ, Rudnick A, Garcia R, MacVean DW. Alphaviruses in Peninusular Malaysia: I. Virus isolations and animal serology. Southeast Asian J Trop Med Public Health. 1978;9(3):317-329.

36. Peiris JS, Dittus WP, Ratnayake CB. Seroepidemiology of dengue and other arboviruses in a natural population of toque macaques (Macaca sinica) at Polonnaruwa, Sri Lanka. J Med Primatol. 1993;22(4):240-245.

37. Cawthon Lang KA. Primate factsheets: Long-tailed macaque (Macaca fascicularis) taxonomy, morphology, \& ecology. http://pin.primate.wisc.edu/ factsheets/entry/long-tailed_macaque/taxon. Updated January 6, 2006. Accessed January 28, 2010.

38. Chastel C. Chikungunya virus: its recent spread to the southern Indian Ocean and Reunion Island (2005-2006) [in French]. Bull Acad Natl Med. 2005;189(8):1827-1835

\title{
Lights on for aminopeptidases in cystic kidney disease
}

\author{
Erwin P. Böttinger
}

Charles R. Bronfman Institute for Personalized Medicine, and Department of Medicine, Mount Sinai School of Medicine, New York, New York.

\begin{abstract}
While erudite cell biologists have for many decades described singular immotile appendages known as primary cilia to be present on most cells in our bodies, cilial function(s) long remained an enigma. Driven largely by an ever increasing number of discoveries of genetic defects in primary cilia during the past decade, cilia were catapulted from a long lasting existence in obscurity into the bright spotlight in cell biology and medicine. The study by O'Toole et al. in this issue of the JCI adds a novel "enzymatic" facet to the rapidly growing information about these little cellular tails, by demonstrating that defects in the XPNPEP3 gene, which encodes mitochondrial and cytosolic splice variants of $X$-prolyl aminopeptidase 3 , can cause nephronophthisis-like ciliopathy. Future studies are in order now to elucidate the cystogenic pathways affected by disrupted enzymatic function of XPNPEP3 in cilia-related cystogenic diseases.
\end{abstract}

Conflict of interest: The author has declared that no conflict of interest exists.

Citation for this article: $J$ Clin Invest. 2010; 120(3):660-663. doi:10.1172/JCI42378.
Based on a flood of recent evidence, primary cilia are now heralded as sensory organelles for detection and transmission of a broad range of cues from the extracellular environment of cells (1), including mechanical and chemical information as diverse as fluid flow in kidneys, mechanical bone deformation, and light and odorant detection (2). By processing such physical and chemical information from the environment into molecular signals in development and postnatal growth and homeostasis, cilia can affect cell differentiation and polarity and cell cycle control (3).

Genetic damage to primary cilia results in a spectrum of perplexing disorders with seemingly disparate manifestations, now classified as ciliopathies. A growing list of bona fide ciliopathies at present includes Bardet-Biedl syndrome (BBS), nephronophthisis (NPHP), and Senior-Loken syndrome (SNLS), just to name a few. Common 